\title{
Preoperative Mental Health May Not Be Predictive of Improvements in Patient-Reported Outcomes Following a Minimally Invasive Transforaminal Lumbar Interbody Fusion
}

\author{
BENJAMIN C. MAYO, MD, ANKUR S. NARAIN, MD, FADY Y. HIJJI, MD, DUSTIN H. MASSEL, MD, \\ DANIEL D. BOHL, MD, MPH, KERN SINGH, MD \\ Department of Orthopaedic Surgery, Rush University Medical Center, Chicago, Illinois
}

\begin{abstract}
Background: Prior literature has associated poor preoperative mental health with inferior patient-reported outcomes (PROs) after spinal procedures. Therefore, the objective of this study was to test for association of preoperative Short Form 12 (SF-12) mental health composite score (MCS) with improvements in Oswestry Disability Index (ODI) and back and leg visual analogue scale (VAS) pain scores after a minimally invasive transforaminal lumbar interbody fusion (MIS TLIF).

Methods: A surgical database of patients who underwent a primary, 1 level MIS TLIF was reviewed. Preoperative SF-12 MCS was tested for association with preoperative ODI, back VAS, and leg VAS. Preoperative MCS was then tested for association with changes in ODI, back VAS, and leg VAS from preoperative to postoperative visits. These tests were conducted using multivariate regression controlling for baseline characteristics and the preoperative score of the PRO being assessed.

Results: A total of 113 patients were included in the analysis. At baseline, higher preoperative MCS was associated with lower preoperative ODI (coefficient: $-0.58, P<.001)$, lower preoperative back VAS $(-0.05, P=.003)$, and lower preoperative leg VAS $(-0.06, P=.003)$. However, there was no association between preoperative MCS and improvement in PROs at any postoperative timepoint $(P>.05)$. The percent of patients achieving a minimum clinically important difference in PROs at 6 months did not differ between the bottom and top MCS halves $(P>.05)$.

Conclusions: The results of this study suggest that better preoperative mental health is associated with lower perceived preoperative disability and decreased severity of back and leg pain. In contrast to other studies, the present study was unable to demonstrate that preoperative mental health is predictive of improvement in PROs at any postoperative timepoint after MIS TLIF.

Level of Evidence: 3

Clinical Relevance: These results suggest that surgeons should exercise care in assuming that patients with poorer preoperative mental health are inferior surgical candidates.

Minimally Invasive Surgery

Keywords: mental health, depression, transforaminal lumbar interbody fusion, TLIF, outcomes, SF-12, ODI, VAS
\end{abstract}

\section{INTRODUCTION}

Lumbar spine surgery is a commonly used treatment for low back pain refractory to conservative management. However, a substantial proportion of patients still may not experience relief of symptoms. In some instances, success rates as low as $65 \%$ have been reported, with reoperation rates near $25 \% .^{1-4}$ Recently, an increasing number of studies have suggested that preoperative psychological and affective symptoms may play a role in a patient's postoperative outcomes. ${ }^{5-10}$ Depression and anxiety are the most common psychosomatic symptoms thought to be associated with poor outcomes after spinal surgery, though other psychiatric and mental health disorders have been reported as negative predictors of success as well. While several studies have concluded that preoperative mental health is correlated with outcomes after spinal procedures, ${ }^{8,9}$ other studies have reported that depression and mental health are not associated with worsened outcomes. ${ }^{11,12}$

The Short Form Health Survey (SF-12) is a concise, validated, and widely used instrument that assesses the global health of patients. ${ }^{13,14}$ The SF-12 is scored in 2 subsections, a physical component score (PCS), which evaluates physical function, and 
a mental component score (MCS), which evaluates emotional health and has been reported to be an acceptable screen for depression. ${ }^{13-16}$ The purpose of this study is to test for an association between preoperative mental health as measured using the SF-12 MCS and changes in patient-reported outcomes (PROs) after a minimally invasive transforaminal lumbar interbody fusion (MIS TLIF).

\section{METHODS AND MATERIALS}

\section{Patient Population}

After institutional review board approval (ORA \#14051301), a prospectively maintained surgical database of consecutive patients who underwent a primary, 1 level MIS TLIF at a single academic institution during 2013-2016 was reviewed. All patients were invited to fill out a SF-12, an Oswestry Disability Index (ODI), and visual analogue scale (VAS) pain scores for back and leg at each clinic appointment (preoperatively, 6 and 12 weeks, and 6 months postoperatively). All surveys were assigned to be completed online before each patient's respective preoperative and postoperative visits. If surveys were not completed before a scheduled visit, patients were instructed to complete the surveys on a touchscreen tablet computer in the office before being evaluated by the surgeon.

Follow up of 6 months was deemed appropriate as previous studies have demonstrated statistically significant improvements in PROs in as few as 3 months postoperatively. ${ }^{17}$ Only patients who completed all 4 PRO surveys at all 4 visits were included in the analysis.

\section{Outcome Measures}

The SF-12 survey is used to assess general health status. In both subsections (PCS and MCS), a higher score indicates better status. The ODI is used to assess overall disability in patients with low back pain. For this measure, a higher score correlates with higher disability, while a lower score is indicative of minimal disability. VAS is a numerical scale ranging from 0 to 10 to assess severity of pain in a specific body region. In this scale, a score of 10 indicates severe pain, while 0 indicates no pain.

For each of the above-reported postoperative outcomes (ODI, back VAS, leg VAS), change from preoperative score was calculated at each postoperative timepoint and tested for association with preoperative MCS as a continuous variable. Addi- tionally, patients in the top half of preoperative MCS scores were compared to patients in the bottom half of preoperative MCS scores regarding achievement of minimum clinically important difference (MCID) in PROs. The cutoff for the top and bottom half of preoperative MCS was a score of 49.0. MCID was assessed for ODI, back VAS, and leg VAS at the 6 month postoperative visit. Based on the MCID values reported in the literature, improvements of $12.8,1.2$, and $1.6^{18,19}$ were used as the MCID for ODI, back VAS, and leg VAS, respectively.

\section{Statistical Methods}

Statistical analysis was performed using Stata/ MP ${ }^{\circledR} 13.1$ for Mac (StataCorp LP, College Station, TX). First, to characterize associations at baseline, preoperative SF-12 MCS was tested for association with preoperative ODI, back VAS, and leg VAS using bivariate regression. Next, preoperative SF-12 MCS was tested for an association with changes in ODI, back VAS, and leg VAS at each postoperative timepoint using multivariate linear regression controlling for patient age, gender, smoking status, obesity, insurance type, Charlson Comorbidity Index $(\mathrm{CCI})$, and the preoperative score of the PRO being assessed. Finally, patients in the top and bottom halves of preoperative MCS were compared using multivariate linear regression controlling for all preoperative characteristics. A $P$ value $<.05$ was used to determine statistical significance.

\section{RESULTS}

A total of 227 consecutive patients underwent a primary, 1 level MIS TLIF during the study time period and were eligible for the study. Of those, 114 patients $(50.2 \%)$ were excluded from this analysis due to not having complete survey data for all preoperative and postoperative visits. As a result, 113 patients with complete survey data for all visits were included in the final analysis. Table 1 describes the baseline characteristics of the study population. Of note, the average age of included patients was $52.8 \pm 12.1$ years.

Figures 1-3 depict the associations of preoperative MCS with preoperative PROs in patients at baseline. Higher preoperative MCS was associated with lower preoperative ODI (coefficient: -0.58 , 95\% CI: -0.82 to $-0.33, P<.001$; Figure 1 ). Similarly, higher preoperative MCS was associated 
Table 1. Baseline characteristics of patient population.

\begin{tabular}{|c|c|}
\hline & $(\mathrm{N}=113)$ \\
\hline Age $($ mean $\pm S D, y)$ & $52.8 \pm 12.1$ \\
\hline \multicolumn{2}{|l|}{$\operatorname{Sex}(n)$} \\
\hline Female & $32.7 \%(37)$ \\
\hline Male & $67.3 \%(76)$ \\
\hline \multicolumn{2}{|l|}{ Insurance (n) } \\
\hline Medicare & $8.9 \%(10)$ \\
\hline Workers' Compensation & $29.2 \%(29)$ \\
\hline Commercial & $62.0 \%(70)$ \\
\hline \multicolumn{2}{|l|}{ Smoking Status (n) } \\
\hline Nonsmoker & $85.0 \%(96)$ \\
\hline Smoker & $15.0 \%(17)$ \\
\hline \multicolumn{2}{|l|}{ Operative Levels (n) } \\
\hline L3-L4 & $3.5 \%(4)$ \\
\hline L4-L5 & $46.0 \%(52)$ \\
\hline L5-S1 & $50.4 \%(57)$ \\
\hline \multicolumn{2}{|l|}{ Body Mass Index (n) } \\
\hline$<30 \mathrm{~kg} / \mathrm{m}^{2}-$ nonobese & $46.9 \%(53)$ \\
\hline$>30 \mathrm{~kg} / \mathrm{m}^{2}-$ Obese & $53.1 \%(60)$ \\
\hline Comorbidity (mean $\pm \mathrm{SD}, \mathrm{CCI})$ & $1.90 \pm 1.80$ \\
\hline Preoperative MCS & $48.0 \pm 11.64$ \\
\hline
\end{tabular}

Abbreviations: CCI, Charlson Comorbidity Index; MCS, mental component score; $\mathrm{SD}$, standard deviation.

with lower preoperative back VAS (coefficient: $-0.05,95 \%$ CI: -0.09 to $-0.02, P=.003$; Figure 2). Finally, higher preoperative MCS was associated with lower preoperative leg VAS (coefficient: -0.06 , 95\% CI: -0.11 to $-0.02, P=.003$; Figure 3 ).

Table 2 provides information regarding the association of preoperative MCS with changes in PROs from preoperative to postoperative visits. MCS was not associated with changes in ODI from the preoperative visit at any of the postoperative visits (6 week: $P=.967 ; 12$ week: $P=.842 ; 6$ month: $P=.344)$. Similarly, preoperative MCS was not associated with changes in back VAS from the preoperative visit at any postoperative visits (6 week: $P=.656 ; 12$ week: $P=.427 ; 6$ month: $P=.075)$. Finally, preoperative MCS was not

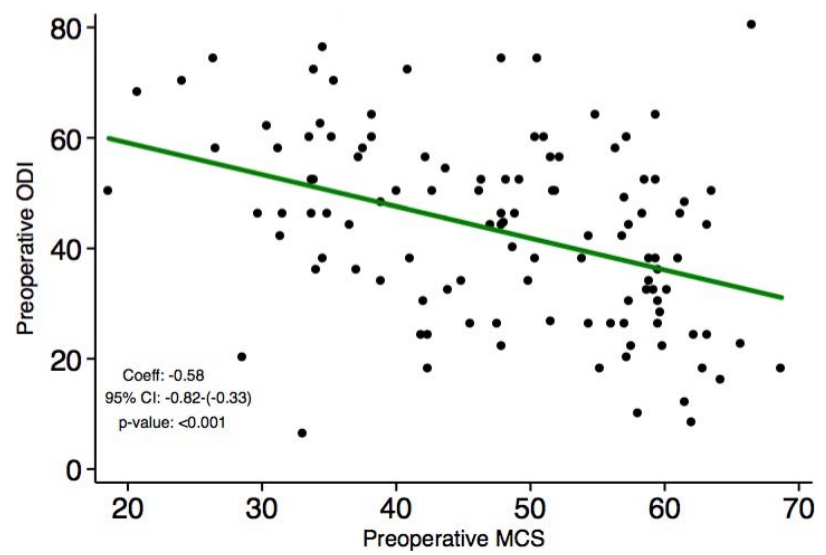

Figure 1. Higher preoperative Short Form 12 mental health composite score (MCS) is associated with lower preoperative Oswestry Disability Index (ODI).

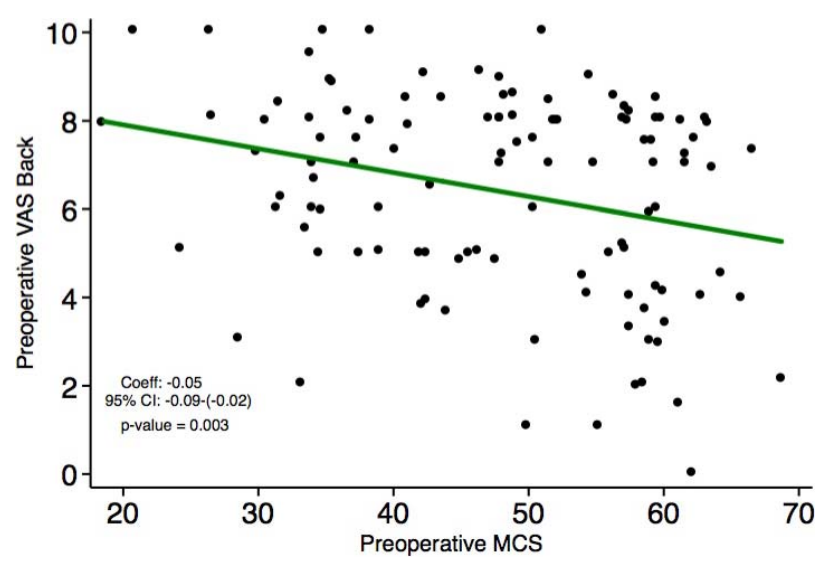

Figure 2. Higher preoperative Short Form 12 mental health composite score (MCS) is associated with lower preoperative visual analogue scale (VAS) back pain.

associated with changes in leg VAS from the preoperative visit at any postoperative visits (6 week: $P=.714 ; 12$ week: $P=.398 ; 6$ month: $P=.414)$.

Table 3 provides information on the achievement of MCID for each of the PROs. The percent of patients achieving MCID did not differ between patients in the bottom and top halves of preoperative MCS for ODI $(73.2 \%$ versus $61.4 \%, P=.362)$, back VAS $(75.0 \%$ versus $80.7 \%, P=.521)$, or leg VAS (67.9\% versus $70.2 \%, P=.657)$.

Table 4 provides information on the preoperative MCS scores and achievement of MCID for each of the PROs. There were no differences in mean preoperative MCS between those patients who achieved MCID and those who did not for ODI $(P=.529)$, back VAS $(P=.171)$, or leg VAS $(P=.235)$.

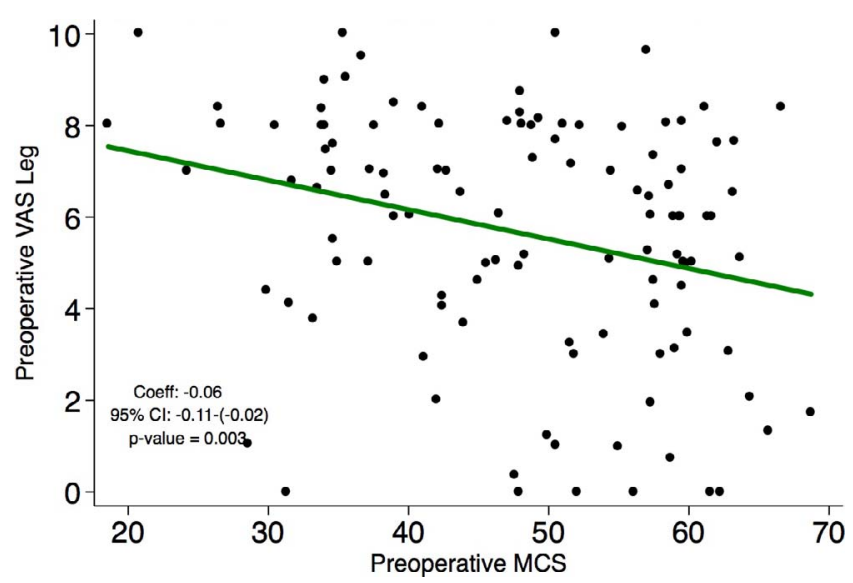

Figure 3. Higher preoperative Short Form 12 mental health composite score (MCS) is associated with lower preoperative visual analogue scale (VAS) leg pain. 
Table 2. Preoperative mental health score association with patient reported outcome measures.

\begin{tabular}{|c|c|c|c|c|c|}
\hline & Mean \pm SD & Change \pm SD & Coefficient $\pm \mathrm{SE}$ & $95 \%$ CI & $P$ Value $^{\mathrm{a}}$ \\
\hline \multicolumn{6}{|l|}{ ODI } \\
\hline Preoperative & $43.0 \pm 16.8$ & - & - & - & - \\
\hline 6 week & $37.4 \pm 19.6$ & $-5.6 \pm 18.9$ & $0.01 \pm 0.15$ & $-0.29-0.30$ & .967 \\
\hline 12 week & $29.7 \pm 17.9$ & $-13.3 \pm 17.2$ & $-0.02 \pm 0.12$ & $-0.26-0.21$ & .842 \\
\hline 6 month & $22.5 \pm 18.0$ & $-20.5 \pm 16.9$ & $-0.11 \pm 0.12$ & $-0.35-0.12$ & .344 \\
\hline \multicolumn{6}{|l|}{ VAS Back } \\
\hline Preoperative & $6.4 \pm 2.3$ & - & - & - & - \\
\hline 6 week & $4.1 \pm 0.5$ & $-2.3 \pm 2.6$ & $0.01 \pm 0.02$ & $-0.03-0.04$ & .656 \\
\hline 12 week & $3.8 \pm 2.6$ & $-2.6 \pm 2.7$ & $-0.01 \pm 0.02$ & $-0.05-0.02$ & .427 \\
\hline 6 month & $3.2 \pm 2.5$ & $-3.1 \pm 3.0$ & $-0.04 \pm 0.02$ & $-0.07-0.01$ & .075 \\
\hline \multicolumn{6}{|l|}{ VAS Leg } \\
\hline Preoperative & $5.7 \pm 2.7$ & - & - & - & - \\
\hline 6 week & $3.1 \pm 3.0$ & $-2.5 \pm 3.4$ & $-0.01 \pm 0.02$ & $-0.06-0.04$ & .714 \\
\hline 12 week & $2.6 \pm 2.6$ & $-3.1 \pm 3.0$ & $0.02 \pm 0.02$ & $-0.02-0.06$ & .398 \\
\hline 6 month & $2.3 \pm 2.5$ & $-3.4 \pm 2.9$ & $-0.02 \pm 0.02$ & $-0.05-0.02$ & .414 \\
\hline
\end{tabular}

Abbreviations: CI, confidence interval; ODI, Oswestry Disability Index; SD, standard deviation; SE, standard error; VAS, visual analogue scale.

${ }^{a} P$ values calculated using multivariate regression controlling for age, gender, smoking status, body mass index category, insurance type, Charlson Comorbidity Index, and preoperative outcome value.

\section{DISCUSSION}

Prior literature has reported several risk factors associated with inferior PROs after spinal surgery. More recently, mental health and depression has taken a central role in this discussion as they have demonstrated the potential to have considerable effects on postoperative outcomes. However, there is debate as to whether spine surgeons should consider screening for mental health issues and depression preoperatively. Given this, the purpose of this study was to assess whether preoperative mental health was predictive of outcomes in our population of patients undergoing MIS TLIF.

The results of this study suggest that preoperative SF-12 MCS is not predictive of changes in ODI or VAS from preoperative to postoperative visits. Accordingly, the half of patients with the lowest mental health and the half of patients with the highest mental health achieved MCID in ODI, back VAS, and leg VAS at similar rates. These results suggest that surgeons should exercise care in assuming that patients with poorer preoperative mental health are inferior surgical candidates.

Table 3. Percent of patients who achieved minimum clinically important difference by mental component score half.

\begin{tabular}{lccc}
\hline & $\begin{array}{c}\text { Bottom Half } \\
(\mathbf{N}=\mathbf{5 6})\end{array}$ & $\begin{array}{c}\text { Top Half } \\
(\mathbf{N}=\mathbf{5 7})\end{array}$ & $\boldsymbol{P}$ Value $^{\mathbf{a}}$ \\
\hline ODI (n) & $73.2 \%(41)$ & $61.4 \%(35)$ & .362 \\
VAS Back (n) & $75.0 \%(42)$ & $80.7 \%(46)$ & .521 \\
VAS Leg (n) & $67.9 \%(38)$ & $70.2 \%(40)$ & .657 \\
\hline
\end{tabular}

Abbreviations: MCS, mental component score; ODI, Oswestry Disability Index; VAS, visual analogue scale.

${ }^{\text {a }} P$ values calculated using multivariate regression controlling for age, gender, smoking status, body mass index category, Charlson Comorbidity Index, and preoperative outcome value.
The results of the present study are in line with those of a study by $\mathrm{Ng}$ et al. that assessed predictors of functional outcomes in 100 patients undergoing posterior lumbar decompression for spinal stenosis. Those authors concluded that neither preoperative depression nor preoperative anxiety were associated with worse postoperative outcomes. ${ }^{11}$ Similarly, Parker et al. determined that depression was not associated with postoperative disability or pain, but did report that depressed patients had a lower rate of return to work after lumbar fusion. ${ }^{20}$ Herron et al. reported that depression, hysteria, hypochondriasis, and other psychosomatic symptoms were not predictive of surgical outcomes for lumbar stenosis. ${ }^{21}$ Finally, in 2 separate studies, Katz et al. demonstrated that preoperative psychological factors were not associated with improvement or satisfaction after decompression with or without fusion. ${ }^{3,12}$

However, the results of the current study also contradict much of the current literature, which

Table 4. Preoperative mental health score association with attainment of minimum clinically important difference in patient-reported outcome measures.

\begin{tabular}{lccc}
\hline & N & Mean MCS \pm SD & $P$ Value \\
\hline ODI & & & .529 \\
$\quad$ MCID not achieved & 37 & $49.8 \pm 10.6$ & \\
$\quad$ MCID achieved & 76 & $47.0 \pm 12.1$ & .171 \\
VAS Back & & & \\
$\quad$ MCID not achieved & 25 & $45.9 \pm 10.8$ & .235 \\
MCID achieved & 88 & $48.6 \pm 11.9$ & \\
VAS Leg & & & \\
$\quad$ MCID not achieved & 35 & $47.6 \pm 11.4$ & \\
$\quad$ MCID achieved & 78 & $48.1 \pm 11.8$ & \\
\hline
\end{tabular}

Abbreviations: MCID, mean clinically important difference; MCS, mental component score; ODI, Oswestry Disability Index; SD, standard deviation; VAS, visual analogue scale.

${ }^{a} P$ values calculated using multivariate regression controlling for age, gender, smoking status, body mass index category, Charlson Comorbidity Index, insurance status, and preoperative outcome value. 
reports that there is an association between mental health issues and inferior outcomes after lumbar spine surgery. ${ }^{8,9,22-29}$ While those studies do report an association between mental health and postoperative outcomes, such findings could not be supported by our data. The greater number of papers reporting the presence of these types of associations may be due to publication bias towards increased reporting of positive results. This phenomenon has been well described: negative results are less likely to be published and, when they are published, are more likely to be reported in lower impact journals than studies with positive results. ${ }^{30-34}$ This bias toward publishing studies that demonstrate an association may be misleading regarding the overall effect of depression and mental health on improvements in PROs after lumbar spine surgery, which may not be as considerable as previously thought.

Despite the lack of an association between preoperative MCS and postoperative changes in ODI and VAS, preoperative MCS was negatively associated with perceived preoperative disability and pain. That is, at baseline, patients with superior preoperative mental health reported less overall perceived disability, back pain, and leg pain before undergoing surgery. Due to the retrospective nature of this study, determination of causality cannot be made; pain and disability may result in worse mental health, or worse mental health may elicit a higher reporting of pain and disability. These results are in line with prior studies that have reported an association between preoperative depression and preoperative pain or disability., 9,35

The present study is not without limitations. First, our cohort size of 113 patients may be underpowered to detect an association between preoperative mental health and changes in PROs. Second, the evaluation of a patient's mental health via SF-12 MCS may not be as effective as using a validated survey specifically designed to assess depression or anxiety. However, SF-12 has been demonstrated to be an effective tool in screening for both recent and active depression and is more widely implemented in orthopaedic practices compared to depression-specific tests. ${ }^{15}$ Third, the follow-up period of 6 months may not adequately capture the long-term trends of PRO improvement after MIS TLIF. However, it has been demonstrated that significant improvements in VAS and ODI scores are detectable at 3 months postoperatively and that these improvements persist at 2 year follow-up points. ${ }^{17}$ Fourth, the assessment of clinically significant changes in PROs was based on reported MCID values from the literature rather than calculated values specific to the study population. As such, accurate determination of the frequency of MCID achievement between cohorts may be limited. Fifth, because this study was performed at a single academic institution with all surgeries being performed by a single surgeon, the results may not be generalizable to other patient populations and locations. Finally, the retrospective nature of this study introduces inherent biases, and as mentioned previously, causality cannot be determined.

\section{CONCLUSIONS}

The present study suggests that preoperative SF12 MCS is not predictive of change in ODI, back VAS, or leg VAS from preoperative to postoperative timepoints. While lower preoperative MCS was associated with increased perceived preoperative disability and pain, patients with worse mental health demonstrate similar improvements in disability and pain after MIS TLIF. Future studies may wish to assess different preoperative variables as a better predictor of improvement after lumbar surgery.

\section{REFERENCES}

1. Hoffman RM, Wheeler KJ, Deyo RA. Surgery for herniated lumbar discs: a literature synthesis. J Gen Intern Med. 1993;8:487-496.

2. Junge A, Dvorak J, Ahrens S. Predictors of bad and good outcomes of lumbar disc surgery. A prospective clinical study with recommendations for screening to avoid bad outcomes. Spine (Phila Pa 1976). 1995;20:460-468.

3. Katz JN, Stucki G, Lipson SJ, et al. Predictors of surgical outcome in degenerative lumbar spinal stenosis. Spine (Phila Pa 1976). 1999;24:2229-2233.

4. Turner JA, Ersek M, Herron L, et al. Surgery for lumbar spinal stenosis. Attempted meta-analysis of the literature. Spine (Phila Pa 1976). 1992;17:1-8.

5. Adogwa O, Parker SL, Shau DN, et al. Preoperative Zung Depression Scale predicts patient satisfaction independent of the extent of improvement after revision lumbar surgery. Spine J. 2013;13:501-506.

6. Chaichana KL, Mukherjee D, Adogwa O, et al. Correlation of preoperative depression and somatic perception scales with postoperative disability and quality of life after lumbar discectomy. J Neurosurg Spine. 2011;14:261-267.

7. Menendez ME, Neuhaus V, Bot AG, et al. Psychiatric disorders and major spine surgery: epidemiology and perioperative outcomes. Spine (Phila Pa 1976). 2014;39:E111-122.

8. Miller JA, Derakhshan A, Lubelski D, et al. The impact of preoperative depression on quality of life outcomes after lumbar surgery. Spine J. 2015;15:58-64.

9. Pakarinen M, Vanhanen S, Sinikallio S, et al. Depressive burden is associated with a poorer surgical outcome among 
lumbar spinal stenosis patients: a 5-year follow-up study. Spine J. 2014;14:2392-2396.

10. Trief PM, Ploutz-Snyder R, Fredrickson BE. Emotional health predicts pain and function after fusion: a prospective multicenter study. Spine (Phila Pa 1976). 2006;31:823-830.

11. Ng LC, Tafazal S, Sell P. The effect of duration of symptoms on standard outcome measures in the surgical treatment of spinal stenosis. Eur Spine J. 2007;16:199-206.

12. Katz JN, Lipson SJ, Brick GW, et al. Clinical correlates of patient satisfaction after laminectomy for degenerative lumbar spinal stenosis. Spine (Phila Pa 1976). 1995;20:1155-1160.

13. Farivar SS, Cunningham WE, Hays RD. Correlated physical and mental health summary scores for the SF-36 and SF12 Health Survey, V.I. Health Qual Life Outcomes. 2007;5:54.

14. Gandek B, Ware JE, Aaronson NK, et al. Crossvalidation of item selection and scoring for the SF-12 Health Survey in nine countries: results from the IQOLA Project. International Quality of Life Assessment. J Clin Epidemiol. 1998;51:1171-1178.

15. Vilagut G, Forero CG, Pinto-Meza A, et al. The mental component of the Short-Form 12 Health Survey (SF-12) as a measure of depressive disorders in the general population: results with three alternative scoring methods. Value Health. 2013;16:564-573.

16. Forero CG, Vilagut G, Adroher ND, et al. Multidimensional item response theory models yielded good fit and reliable scores for the Short Form-12 questionnaire. J Clin Epidemiol. 2013;66:790-801.

17. Perez-Cruet MJ, Hussain NS, White GZ, et al. Qualityof-life outcomes with minimally invasive transforaminal lumbar interbody fusion based on long-term analysis of 304 consecutive patients. Spine (Phila Pa 1976). 2014;39:E191-198.

18. Carreon LY, Bratcher KR, Canan CE, et al. Differentiating minimum clinically important difference for primary and revision lumbar fusion surgeries. J Neurosurg Spine. 2013;18:102106.

19. Copay AG, Glassman SD, Subach BR, et al. Minimum clinically important difference in lumbar spine surgery patients: a choice of methods using the Oswestry Disability Index, Medical Outcomes Study questionnaire Short Form 36, and pain scales. Spine J. 2008;8:968-974.

20. Parker SL, Godil SS, Zuckerman SL, et al. Extent of preoperative depression is associated with return to work after lumbar fusion for spondylolisthesis. World Neurosurg. 2015;83:608-613.

21. Herron LD, Turner J, Clancy S, et al. The differential utility of the Minnesota Multiphasic Personality Inventory. A predictor of outcome in lumbar laminectomy for disc herniation versus spinal stenosis. Spine (Phila Pa 1976). 1986;11:847-850.

22. Adogwa O, Parker SL, Shau DN, et al. Preoperative Zung Depression Scale predicts outcome after revision lumbar surgery for adjacent segment disease, recurrent stenosis, and pseudarthrosis. Spine J. 2012;12:179-185.

23. Adogwa O, Verla $\mathrm{T}$, Thompson $\mathrm{P}$, et al. Affective disorders influence clinical outcomes after revision lumbar surgery in elderly patients with symptomatic adjacent-segment disease, recurrent stenosis, or pseudarthrosis: clinical article. $J$ Neurosurg Spine. 2014;21:153-159.

24. Alvin MD, Miller JA, Sundar S, et al. The impact of preoperative depression on quality of life outcomes after posterior cervical fusion. Spine J. 2015;15:79-85.
25. Andersen T, Christensen FB, Bunger C. Evaluation of a Dallas Pain Questionnaire classification in relation to outcome in lumbar spinal fusion. Eur Spine J. 2006;15:1671-1685.

26. Arpino L, Iavarone A, Parlato C, et al. Prognostic role of depression after lumbar disc surgery. Neurol Sci. 2004;25:145-147.

27. Block AR, Ohnmeiss DD, Guyer RD, et al. The use of presurgical psychological screening to predict the outcome of spine surgery. Spine J. 2001;1:274-282.

28. LaCaille RA, DeBerard MS, Masters KS, et al. Presurgical biopsychosocial factors predict multidimensional patient: outcomes of interbody cage lumbar fusion. Spine $J$. 2005;5:71-78.

29. McKillop AB, Carroll LJ, Battie MC. Depression as a prognostic factor of lumbar spinal stenosis: a systematic review. Spine J. 2014;14:837-846.

30. Chalmers I. Underreporting research is scientific misconduct. JAMA. 1990;263:1405-1408.

31. Easterbrook PJ, Berlin JA, Gopalan R, et al. Publication bias in clinical research. Lancet. 1991;337:867-872.

32. Dwan K, Altman DG, Arnaiz JA, et al. Systematic review of the empirical evidence of study publication bias and outcome reporting bias. PLoS One. 2008;3:e3081.

33. Liebeskind DS, Kidwell CS, Sayre JW, et al. Evidence of publication bias in reporting acute stroke clinical trials. Neurology. 2006;67:973-979.

34. Littner Y, Mimouni FB, Dollberg S, et al. Negative results and impact factor: a lesson from neonatology. Arch Pediatr Adolesc Med. 2005;159:1036-1037.

35. Chapin L, Ward K, Ryken T. Preoperative depression, smoking, and employment status are significant factors in patient satisfaction after lumbar spine surgery. Clin Spine Surg. 2017; 30(6):E725-E732.

Disclosures and COI: No funds were received in support of this work. No benefits in any form have been or will be received from any commercial party related directly or indirectly to the subject of this manuscript. The manuscript submitted does not contain information about medical device(s) or drug(s). No FDA device or drug status to report. Unlabeled or investigational uses of any commercial product or device have not been discussed. Institutional review board approval was granted for this study (ORA Number 14051301).

Correspondence Author: Kern Singh, MD, Department of Orthopaedic Surgery, Rush University Medical Center, 1611 W. Harrison St, Suite \#300, Chicago, IL 60612. Phone: (312) 432-2373; Fax: (708) 409-5179; E-mail: Kern.singh@rushortho.com.

Published 29 February 2020

This manuscript is generously published free of charge by ISASS, the International Society for the Advancement of Spine Surgery. Copyright (c) 2020 ISASS. To see more or order reprints or permissions, see http://ijssurgery.com. 